\title{
Potential Association of EEF1A Dimethylation at Lysine 55 in the Basal Area of Helicobacter Pylori- Eradicated Gastric Mucosa with the Risk of Gastric Cancer: A Retrospective Observational Study
}

Yuka Hirashita ( $\nabla$ not-minamino@oita-u.ac.jp )

Oita University

Masahide Fukuda

Oita University

Masaaki Kodama

Oita University

Yoshiyuki Tsukamoto

Oita University

Tadayoshi Okimoto

Oita University

Kazuhiro Mizukami

Oita University

Yoshinari Kawahara

Oita University

Yasuhiro Wada

Oita University

Kazumi Togo

Oita University

Kensuke Fukuda

Oita University

Kazuhisa Okamoto

Oita University

Ryo Ogawa

Oita University

Osamu Matsunari

Oita University

Koichi Honda

Oita University

Kazunari Murakami

Oita University 


\section{Research Article}

Keywords: Atrophic gastritis, Gastric cancer, Risk factor, Methylation

Posted Date: November 29th, 2021

DOI: https://doi.org/10.21203/rs.3.rs-1072695/v1

License: (c) (1) This work is licensed under a Creative Commons Attribution 4.0 International License. Read Full License 


\section{Abstract \\ Background}

Although eradication therapy for chronic Helicobacter pylori reduces the risk of gastric cancer (GC), its effectiveness is incomplete. Therefore, it is critically important to identify those patients who remain at high risk after $H$. pylori eradication therapy. Accumulation of protein methylation is strongly implicated in cancer, and a recent study showed that dimethylation of eEF1A lysine 55 (eEF1AK55me2) promotes carcinogenesis in vivo. We aimed to investigate the relationship between eEF1A dimethylation and $H$. pylori status in gastric mucosa and to reveal potential downstream molecules of eEF1A dimethylation in H. pylori-eradicated mucosa.

\section{Methods}

Records of 115 patients ( 11 H. pylori-negative, 29 H. pylori-positive, 75 post-eradication patients) who underwent upper gastrointestinal endoscopy were retrospectively reviewed. The eEF1A dimethyl level was evaluated in each functional cell type of gastric mucosa by immunofluorescent staining. We also investigated the relationship between eEF1AK55me2 downregulation by CRISPR/Cas9-mediated deletion of Mettl13, which is known as a dimethyltransferase of eEF1AK55me2.

\section{Results}

The level of eEF1A dimethylation significantly increased in the surface and basal areas of $H$. pyloripositive mucosa compared with -negative mucosa (surface, $p=0.0031$; basal, $p<0.0001$ ). The eEF1A dimethyl levels in the surface area were significantly reduced by eradication therapy $(p=0.005)$, but those in the basal area were maintained even after eradication therapy. Multivariate analysis revealed that high dimethylation of eEF1A in the basal area of the mucosa was the independent factor related to GC incidence (odds ratio $=3.6611,95 \%$ confidence interval $=1.0350-12.949, p=0.0441$ ). We also showed the relationship between eEF1A dimethylation and expressions of reprogramming factors Oct4 and Nanog by immunohistochemistry and in vitro genome editing experiments.

\section{Conclusions}

The results indicated that $H$. pylori infection potently induced eEF1A dimethylation in gastric mucosa. The accumulation of dimethyl-eEF1A in the basal area of the mucosa might contribute to GC risk via regulation of reprograming factors in $\mathrm{H}$. pylori-eradicated gastric mucosa.

\section{Introduction}


Helicobacter pylori infection, which causes atrophic gastritis, has been defined as a sequence of histological events that confers an increasing risk of malignant transformation [1, 2]. Reductions in primary and metachronous gastric cancer (GC) are expected with $H$. pylori eradication, whereas eradication alone does not completely eliminate GC risk in patients with atrophic gastric mucosa as precancerous lesions [3, 4]. Therefore, even after eradication therapy, identification of patients with high risk of GC is necessary to improve patient outcomes via an effective follow-up strategy.

In previous studies, accumulations of methylation in gastric mucosa after $H$. pylori eradication therapy were correlated with GC risk [5-7]. Most of these reports addressed only epigenetic biomarkers, such as DNA or histone methylation, in regard to the risk of GC in patients with chronic gastritis $[8,9]$. However, it was recently revealed that hundreds and likely thousands of proteins are also methylated at lysine, which leads to upregulation of cellular pathways such as those for growth signaling and response to damage from chronic inflammation [10-12]. Eukaryotic elongation factor $1 A(e E F 1 A)$ is one of the evolutionarily conserved and fundamental non-ribosomal components of the translational machinery [13]. The expression or upregulation of eEF1A has been reported to be associated with cancer development and invasion in ovarian, breast, lung, prostate, hepatic, and pancreatic cancers [14-16, 17]. Additionally, a recent study showed that dimethylation of eEF1Al lysine 55 (eEF1AK55me2) leads to its activation and utilization to increase translational output and promote carcinogenesis in vivo [12].

Although methylation is recognized as a key mechanism in cancer development in atrophic gastric mucosa $[5,18]$, how lysin methylation is maintained in atrophic gastric mucosa and contributes to GC development remains mostly unclear.

The aim of this study was to clarify the association between the dimethylation of eEF1A, an active GTPase form, and $H$. pylori infection status and GC incidence in gastric mucosa. Additionally. we investigated how dimethylated eEF1A contributes to GC development after eradication in gastric mucosa by focusing on the reprogramming factors Oct4 and Nanog.

\section{Materials And Methods}

\section{Study subjects}

We retrospectively investigated the mucosa of the middle portion of the greater curvature of the gastric corpus, approximately $8 \mathrm{~cm}$ from the gastric cardia, in patients who underwent upper gastrointestinal endoscopy with biopsy at Oita University Hospital, Japan, between January 2001 and December 2018. The biopsy site "B2", which is recommended by the Updated Sydney System, is assumed to be best suited for evaluating the histological status of the fundic glands [19]. H. pylori infection was determined by culture, histology, and rapid urease test. In H. pylori-negative patients, we additionally confirmed the lack of endoscopic atrophy and histologic gastritis.

\section{Histological analysis}


Specimens were collected endoscopically from the greater curvature of the gastric middle bodies. Biopsy specimens were immediately fixed in $10 \%$ neutral buffered formalin for $24 \mathrm{~h}$ and embedded in paraffin

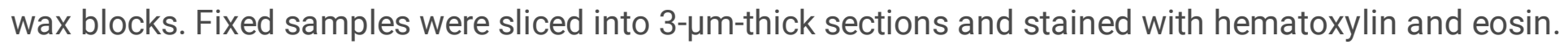
Degrees of inflammation, activity, atrophy, and intestinal metaplasia were scored according to the Updated Sydney System (0, none; 1, weak; 2, moderate; 3, marked) [19].

\section{Immunofluorescent staining}

Anti-MUC5AC (1:50, Leica Biosystems), $\mathrm{H}^{+} / \mathrm{K}^{+}$ATPase (1:100; Medical \& Biological Laboratories), MUC6 (1:200; BioRad), and PG1 (1:200; Abnova) antibodies were used for immunostaining to differentiate between cell types in the fundic glands. We performed immunohistochemistry using anti-dimethyleEF1AK55me2 antibody (1:400; ABclonal Technology), Nanog (1:10000; Cell Signaling Technology), and Oct4 (1:100; Santa Cruz Biotechnology, Santa Cruz, CA, USA). All biopsy materials were fixed in 10\% buffered formalin for $24 \mathrm{~h}$ and then embedded in paraffin. After deparaffinization and subsequent rehydration to remove xylene, endogenous peroxidases were inactivated with $3 \%$ hydrogen peroxide solution (Wako, Osaka, Japan). Antigen retrieval was performed at pH 6.0. Next, the sections were incubated overnight with monoclonal anti-MUC5AC, MUC6, $\mathrm{H}^{+} / \mathrm{K}^{+} \mathrm{ATPase}, \mathrm{PG} 1$, and dimethyl-eEF1A primary antibodies, followed by incubation with secondary antibodies (1:1000; Alexa Fluor R488 and 594) at room temperature for $2 \mathrm{~h}$. After washing, images were captured with a Keyence BZ-9000 microscope (Keyence, Osaka, Japan).

\section{Evaluation of dimethyl eEF1A level}

Dimethylation levels of eEF1A were evaluated on surface, middle, and basal areas of gastric glands, which were identified by morphological appearance or immunostaining with antibodies for specific markers MUC5AC, $\mathrm{H}+/ \mathrm{K}+$ ATPase, and PG1, respectively. After immunofluorescence staining with antidimethyl-eEF1A antibody, the mean fluorescent intensities of these areas were quantified using the region of interest (ROI) tool in ImageJ software. Multiple ROIs were set for each area based on marker immunofluorescence in one image, and several images at $4 \times$ magnification were used for each patient. Then, the dimethylation level of eEF1A for each area in each patient was expressed as an average of multiple mean fluorescent intensities from the ROIs.

\section{Statistical analysis}

We investigated the relationship between the clinical factors and dimethyl-eEF1A level of atrophic gastritis mucosa using univariate and multivariate analysis. All variables are expressed as means \pm standard deviation for continuous data (Table 2). Prior to analysis, continuous data were divided into two groups using average values. Levels of eEF1A dimethylation were compared between groups using analysis of variance or analysis of covariance (for age-adjusted data), followed by the Tukey test. Univariate analyses were performed using the Student $t$-test or the Tukey test for continuous variables and chi-squared test for categorical variables. The results of the multivariate logistic regression analysis are expressed as adjusted odds ratios (aOR) with 95\% confidence intervals (CI). A $p$-value $<0.05$ was 
considered to indicate statistical significance. All statistical analyses were performed using with $\mathrm{JMP}^{\circledR} 11$ (SAS Institute Inc, Cary, NC, USA).

\section{Cell culture and CRISPR/Cas9}

TMK-1 was provided by Dr. H. Ito (Tottori Prefectural Kousei Hospital, Tottori, Japan) with permission from an original provider, Dr. A. Ochiai (National Cancer Center, Kashiwa, Japan). Cells were cultured in RPMI1640 (Sigma) with 10\% fetal bovine serum. We established Mettl13-knockout TMK-1 cells using the CRISPR-Cas9 genome editing system and generated stable Mkettl13 knockdown TMK-1 cells. Briefly, a single guide (sgRNA) targeting Mettl13 (5'-AAGAAAGCTTTCGAGTGGTATGG -3') was cloned into a Cas9expressing plasmid (pSpCas9(BB)-2A-Puro(PX450); Addgene plasmid \#62988), and the plasmid was transfected into TMK-1 cells, which was subsequently cloned after a 2-day treatment with $2 \mu \mathrm{g} / \mathrm{mL}$ puromycine. Knockout of Mett113 was confirmed by Western blotting.

\section{Western blotting}

Western blotting was performed as described previously [20]. Cells were lysed on ice for 20 min in SDSmodified RIPA buffer containing protease and phosphatase inhibitor cocktails (cOmplete Mini; Roche Diagnostics, Mannheim, Germany) (PhosSTOP EASYpack; Roche Diagnostics) and then centrifuged at $4^{\circ} \mathrm{C}$ at $15,000 \mathrm{rpm}$ for $20 \mathrm{~min}$. The resulting cell lysates ( $20 \mu \mathrm{g}$ each) were boiled with Laemmli sample buffer and subjected to SDS-PAGE. The samples were transferred to a polyvinylidene difluoride membrane (Merk Millipore, Darmstadt, Germany), which was blocked for $1 \mathrm{~h}$ in Block Ace (DS Pharma, Osaka, Japan) at room temperature and then incubated overnight at $4{ }^{\circ} \mathrm{C}$ with primary antibodies against METTL13 (1:1000; Bethyl Laboratories), dimethyl-eEF1A (1:1000; ABclonal Technology), eEF1A(1:1000; ABclonal Technology), Nanog (1:1000; Abcam), Oct4 (1:1000, Abcam), and GAPDH (1:1000, Santa Cruz Biotechnology). After washing with TBS containing $0.1 \%$ Tween 20 , the membranes were incubated for 1 $\mathrm{h}$ at room temperature with appropriate secondary antibodies, followed by rewashing [20]. Finally, the signals were detected using an ECL Western blotting analysis system (GE Healthcare, Piscataway, NJ, USA) in accordance with the manufacturer's instructions.

\section{Results}

We retrospectively investigated the gastric corpus mucosa from 115 subjects $(11 \mathrm{H}$. pylori-negative, $29 \mathrm{H}$. pylori-positive, and 75 post-eradication patients). The characteristic of the patients are provided in Table 1. Updated Sydney System scores for inflammation, activity, atrophy, and intestinal metaplasia (IM) are shown in Table 2. The dimethyl level of eEF1A of each gland area identified morphologically or by immunostaining for specific markers (surface, MUC5A; middle, H+/K+ATPase; and basal, PG1) was evaluated (Suppl. Figure 1 and Table 2). In the middle area, dimethyl-eEF1A was not detected in MUC6positive cells but only in $\mathrm{H}+/ \mathrm{K}+$ ATPase-positive cells (Suppl. Figure 1 ).

\section{Difference in eEF1A dimethylation of gastric mucosa by $H$. pylori infection status}


To investigate the association between $H$. pylori infection/eradication and eEF1A dimethylation in gastric mucosa, we first compared the dimethylation levels of eEF1A in each area (MUC5AC-, $\mathrm{H}^{+/} \mathrm{K}^{+}$ATPase-, and PG1-positive cells) between the three groups: H. pylori-negative, H. pylori- positive, and post-eradicated patients (Figure 1a). H. pylori-positive patients showed higher eEF1A dimethylation than H. pylorinegative patients in the surface and basal areas of the gastric mucosa (surface: $16.91 \pm 6.42$ vs. $27.95 \pm 10.95, p=0.0003$; basal: $14.03 \pm 4.88$ vs. $29.31 \pm 6.19, p<0.0001)$. These results suggested that dimethylation of eEF1A is associated with $H$. pylori infection in gastric mucosa.

The dimethylated eEF1A that was caused by $H$. pylori infection was effectively reduced in the surface area (MUC-5AC-positive area) of post-eradicated mucosa $(p=0.0050)$ (Figure 1b). However, $H$. pylori eradication therapy did not affect eEF1A dimethylation in the basal area (PG-1-positive area) (Figure 1b). Because there were age differences between these three groups (Table 1), we also calculated the age-adjusted levels of eEF1A dimethylation and compared them between the groups using analysis of covariance with the Tukey test. We found the same tendency in both the crude and ageadjusted models, indicating that age-related differences in methylation did not account for the results (Figure 1b). These results suggested that the dimethylation of eEF1A in the basal level remained high in atrophic gastric mucosa even after $H$. pylori eradication therapy.

\section{Univariate and multivariate analyses of GC and eEF1A dimethylation in basal area of gastric mucosa}

As the accumulation of methylation is known to be one of the important factors in gastric carcinogenesis $[21,22]$, we investigated whether dimethylation of eEF1A in the basal area could cause a risk of cancer in post-eradicated atrophic gastritis mucosa. The influence of known potential risk factors for GC (age, sex, period after eradication therapy, and histological features) and eEF1A dimethyl level in basal mucosa were analyzed in $75 \mathrm{H}$. pylori eradication patients, which included in part those with a history of GC treatment. Univariate analyses showed that severe histological atrophy and high eEF1A dimethylation of the basal mucosal area were the risk factors for GC (Figure 2 and Table 3). Multivariate analysis revealed that not only histological atrophic levels $(\mathrm{aOR}=9.9493,95 \% \mathrm{Cl}=1.7096-57.901, p=0.0106)$ but also high dimethylation of eEF1A in basal mucosal areas (aOR=3.6611, 95\% $\mathrm{Cl}=1.0350-12.949, p=0.0441)$ were the independent factors related to GC incidence (Table 3 ).

\section{Accumulation of eEF1A dimethylation and metaplasia in gastric mucosa}

Metaplastic lineages have been associated with various injurious scenarios in atrophic gastritis mucosa. We investigated eEF1A dimethylation in the area of IM $(n=11)$ of eradicated mucosa. There was no significant accumulation in eEF1A dimethylation in the IM area compared with other gastric gland cells (mean: $22.01 \pm 8.86$, Table 2). Although IM is considered an important step in the pathogenesis of GC in atrophic gastric mucosa, there was no significant increase in eEF1A dimethylation in the IM area in eradicated patients with $\mathrm{GC}$ incidence (GC(-), $\mathrm{n}=7$ and $\mathrm{GC}(+), \mathrm{n}=4)$ (Figure 3 ).

Association of eEF1A dimethylation with the expressions of reprogramming factors Oct4 and Nanog 
In recent reports, chief cells have been identified as a cell capable of reprogramming and metaplastic response to parietal cell damage in atrophic gastric mucosa [23, 24]. Octamer-binding transcription factor 4 (OCT4), and Nanog lie in the core of the transcriptional network that controls stem cell pluripotency [25]. Therefore, we investigated whether the expressions of Oct4 and Nanog correlate with dimethylated eEF1A in post-eradicated mucosa. As shown in Figure $4 a$ and $b$, the proportion of Oct4- or Nanog-positive cells in eradicated mucosa was significantly higher in patients with high eEF1A dimethylation. To investigate whether dimethylated eEF1A contributes to the expressions of Oct4 and Nanog in gastric cells, we next deleted Mettl13, which is the physiologic eEF1A lysine 55 dimethyltransferase [12], in a TMK-1 cell using the CRISPR/Cas9 system and analyzed the effect on the expressions of Oct4 and Nanog. As shown in Figure 4c, knockout of Mettl13 led to a decrease in the eEF1A dimethylation level in TMK-1 cells. Interestingly, reduction of dimethylated eEF1A downregulated the expressions of Oct4 and Nanog in TMK1 cells (Figure 4d). These results suggest that accumulation of dimethylated eEF1A in the basal area of eradicated mucosa is associated with the expression of Oct4 and Nanog, which control reprogramming of various types of differentiated cells in response to chronic damage induced by $\mathrm{H}$. pylori infection.

\section{Discussion}

The presence of H. pylori, a well-known inducer of chronic inflammation and atrophy, is reported to be associated with aberrant DNA methylation in gastric mucosa [5]. However, it remains unknown whether $H$. pylori infection also induces aberrant methylation at the protein level, except for histone modification. In this study, we immunohistochemically analyzed the association of $H$. pylori status with the distribution of dimethylated eEF1A, whose function in tumorigenicity has been characterized recently by Liu et al. [12]. This is first report, to our knowledge, to spatially show the impact of $H$. pylori infection on the level of eEF1A dimethylation in gastric mucosa.

Our results showed that the levels of eEF1A dimethylation in gastric mucosa were different according to H. pylori status (negative, positive, and post-eradication therapy). eEF1A dimethylation of surface and basal cell areas was higher in $H$. pylori-positive than $H$. pylori-negative patients, suggesting that $H$. pylori infection induces eEF1A dimethylation of surface and basal cells in gastric mucosa. Indeed, eEF1A dimethylation in $\mathrm{H}$. pylori-negative mucosa with adenocarcinoma of the fundic gland type, which is known to be unrelated to $H$. pylori infection, was comparable to that in $H$. pylori-negative and cancer-free mucosa (data not shown), further supporting our hypothesis that $H$. pylori infection might cause aberrant protein methylation.

Eradication of $H$. pylori infection has been reported to reduce the risk of GC among asymptomatic patients in high-risk countries [4]. However, long-term studies from Japan revealed that eradication therapy cannot reduce the risk of GC completely $[2,26,27]$. In the present study, we focused on a subset of atrophic gastritis patients who showed high dimethylation of eEF1A in the basal area of $\mathrm{H}$. pylorieradicated mucosa and found that highly dimethylated eEF1A in PG1-positive cells, chief cells in the basal area of the gastric mucosa, correlated with GC incidence in the post-eradicated gastric mucosa. Unlike the basal area of $H$. pylori-eradicated gastric mucosa, the eEF1A dimethylation occurring in the 
surface area (MUC5AC-positive cells) was strongly associated with H. pylori eradication therapy, i.e., the level of eEF1A dimethylation in the surface area were significantly reduced after eradication regardless of the incidence of GC. Considering that the cell lineages in gastric mucosa are known to vary greatly in their life spans, from 3 to 5 days for surface cells to several months for basal chief cells [28], these different life spans among gastric-cell lineages might cause specific accumulation of dimethylated eEF1A in the basal area even after eradication therapy. In our results, eEF1A dimethylation in the basal area of eradicated mucosa did not show significant reduction even after long-term monitoring following $\mathrm{H}$. pylori eradication therapy in patients with GC incidence (follow-up periods of $<3.5$ versus $\geq 3.5$ years, $p=0.3908$ ). Although data from further long-term monitoring after eradication therapy is needed, the level of dimethylated eEF1A could be a potential marker for GC risk during the follow-up of $H$. pylori-eradicated patients.

Although the severity of atrophic damage is already known to be strongly associated with carcinogenesis in gastric mucosa [29], the pathogenic mechanism of how H. pylori infection induces GC has remained a challenging question for decades. Consistent with previous studies, the present study also showed that the patients with severe atrophic change have GC incidence (Table 3). Additionally, we confirmed that eEF1A dimethylation in the basal area of the gastric mucosa is also an independent factor of GC incidence. Previous studies have also shown that damage to the gastric mucosa, including $\mathrm{H}$. pyloriinduced oxyntic atrophy, initiates mucosal metaplasia and the reprogramming and proliferation of basal cells, and these responses are associated with carcinogenesis in atrophic gastric mucosa [23, 30, 31]. Although our results indicated no significant increase in dimethylated eEF1A in the metaplasia area in eradicated gastric mucosa with GC incidence, we showed an association between the expressions of Oct4 and Nanog, which are known as crucial reprogramming factors, and eEF1A dimethylation in the basal area of eradicated gastric mucosa. Additionally, we also created a dimethyl-eEF1A-induced increase in the expression of Oct4 and Nanog in TMK-1 cells. Self-renewing progenitor and differentiating cells expressing reprograming factors may be a target for cancer initiation and progression through the induction of symmetrical cell division [32]. These results suggest that high eEF1A dimethylation in the basal area of eradicated mucosa could not only be a useful marker for GC risk but also might contribute to the development of gastric lesions, such as metaplasia and cancer, via regulation of Oct4 and Nanog. Further molecular functional analysis of eEF1A dimethylation will lead to elucidation of the mechanism of carcinogenesis in H. pylori-eradicated gastric mucosa.

The present study has several limitations. First, this was a retrospective observational study performed at a single center and only included patients who underwent upper gastrointestinal endoscopy for abdominal symptoms or other medical problems reported. Most participants had primary disease; therefore, bias may have occurred. Next, our study contained only 11 patients with IM, 4 of whom had a history of GC treatment (Table 3). Dimethyl-eEF1A was not detected in the MUC6-positive cells, so we therefore focused only on the fundic gland area. Because a recent study showed that alterations in methylation were observed in regions of IM [33], further prospective studies with larger numbers of samples including IM regions in the antrum area of the gastric mucosa may lead to the discovery of new pathological mechanisms of carcinogenesis in eradicated gastric mucosa. 
In conclusion, the present results indicated that $H$. pylori infection induces dimethylation of eEF1A in the surface and basal areas of gastric mucosa. Especially, the dimethylation level of eEF1A in the basal area of gastric mucosae may be associated with the expression of reprogramming factors and GC risk in postH. pylori-eradicated mucosa.

\section{Declarations}

\section{Acknowledgments}

We would like to thank Mr. Yoshiyuki Tsukamoto, Ms. Kanako Ito, and Ms. Yoko Kudo for their excellent assistance with the experiments.

\section{Author contributions}

All authors have approved the final version of the article. Conceptualization: $\mathrm{YH}$; Formal analysis and investigation: $\mathrm{YH}, \mathrm{MF}$, and $\mathrm{MK}$; Writing - original draft preparation: $\mathrm{YH}$; Writing - review and editing: KMizukami, YT, KH, TO, MK; Resources: YK, YW, KT, KF, KO, RO, and OM; Supervision: KMurakami.

\section{Corresponding author}

Correspondence to Yuka Hirashita.

\section{Ethic approval and consent to participate}

This study was approved by the ethics committee of Oita University Faculty of Medicine (approval number: 1541). All procedures were carried out according to the principles outlined in the Declaration of Helsinki. Written informed consent was obtained from the patients prior to study participation.

\section{Consent for publication}

Not applicable.

\section{Conflict of interest}

The authors declare that they have no conflict of interest.

\section{Availability of data and materials}

The datasets in this study available from the corresponding author on reasonable request.

\section{References}

1. Malfertheiner P, Megraud F, O'Morain CA, Atherton J, Axon AT, Bazzoli F, Gensini GF, Gisbert JP, Graham DY, Rokkas T et al: Management of Helicobacter pylori infection-the Maastricht IV/ Florence Consensus Report. Gut 2012, 61(5):646-664. 
2. Murakami K, Kodama M, Nakagawa Y, Mizukami K, Okimoto T, Fujioka T: Long-term monitoring of gastric atrophy and intestinal metaplasia after Helicobacter pylori eradication. Clin J Gastroenterol 2012, 5(4):247-250.

3. Fuccio L, Zagari RM, Eusebi LH, Laterza L, Cennamo V, Ceroni L, Grilli D, Bazzoli F: Meta-analysis: can Helicobacter pylori eradication treatment reduce the risk for gastric cancer? Annals of internal medicine 2009, 151(2):121-128.

4. Lee YC, Chiang TH, Chou CK, Tu YK, Liao WC, Wu MS, Graham DY: Association Between Helicobacter pylori Eradication and Gastric Cancer Incidence: A Systematic Review and Meta-analysis. Gastroenterology 2016, 150(5):1113-1124 e1115.

5. Maekita T, Nakazawa K, Mihara M, Nakajima T, Yanaoka K, Iguchi M, Arii K, Kaneda A, Tsukamoto T, Tatematsu $\mathrm{M}$ et al: High levels of aberrant DNA methylation in Helicobacter pylori-infected gastric mucosae and its possible association with gastric cancer risk. Clinical cancer research : an official journal of the American Association for Cancer Research 2006, 12(3 Pt 1):989-995.

6. Nakajima T, Maekita T, Oda I, Gotoda T, Yamamoto S, Umemura S, Ichinose M, Sugimura T, Ushijima T, Saito D: Higher methylation levels in gastric mucosae significantly correlate with higher risk of gastric cancers. Cancer epidemiology, biomarkers \& prevention : a publication of the American Association for Cancer Research, cosponsored by the American Society of Preventive Oncology 2006, 15(11):2317-2321.

7. Shiotani A, Murao T, Uedo N, lishi H, Yamanaka Y, Kamada T, Kusunoki H, Inoue K, Haruma K: Eradication of $\mathrm{H}$. pylori did not improve abnormal sonic hedgehog expression in the high risk group for gastric cancer. Digestive diseases and sciences 2012, 57(3):643-649.

8. Chen Y, Ren B, Yang J, Wang H, Yang G, Xu R, You L, Zhao Y: The role of histone methylation in the development of digestive cancers: a potential direction for cancer management. Signal transduction and targeted therapy 2020, 5(1):143.

9. Chan $A O$, Rashid $A: C p G$ island methylation in precursors of gastrointestinal malignancies. Current molecular medicine 2006, 6(4):401-408.

10. Scoumanne A, Chen X: Protein methylation: a new regulator of the p53 tumor suppressor. Histol Histopathol 2009, 23(9).

11. Poulard C, Corbo L, Romancer ML: Protein arginine methylation/demethylation and cancer. Oncotarget 2016, 7(41).

12. Liu S, Hausmann S, Carlson SM, Fuentes ME, Francis JW, Pillai R, Lofgren SM, Hulea L, Tandoc K, Lu $\mathrm{J}$ et al: METTL13 Methylation of eEF1A Increases Translational Output to Promote Tumorigenesis. Cell 2019, 176(3):491-504 e421.

13. Negrutskii BS, El'skaya AV: Eukaryotic translation elongation factor 1 alpha: structure, expression, functions, and possible role in aminoacyl-tRNA channeling. Progress in nucleic acid research and molecular biology 1998, 60:47-78.

14. Tomlinson VA, Newbery HJ, Wray NR, Jackson J, Larionov A, Miller WR, Dixon JM, Abbott CM: Translation elongation factor eEF1A2 is a potential oncoprotein that is overexpressed in two-thirds of 
breast tumours. BMC cancer 2005, 5:113.

15. Li R, Wang H, Bekele BN, Yin Z, Caraway NP, Katz RL, Stass SA, Jiang F: Identification of putative oncogenes in lung adenocarcinoma by a comprehensive functional genomic approach. Oncogene 2006, 25(18):2628-2635.

16. Yang S, Lu M, Chen Y, Meng D, Sun R, Yun D, Zhao Z, Lu D, Li Y: Overexpression of eukaryotic elongation factor 1 alpha-2 is associated with poorer prognosis in patients with gastric cancer. Journal of cancer research and clinical oncology 2015, 141(7):1265-1275.

17. Duanmin H, Chao X, Qi Z: eEF1A2 protein expression correlates with lymph node metastasis and decreased survival in pancreatic ductal adenocarcinoma. Hepato-gastroenterology 2013, 60(124):870-875.

18. Asada K, Nakajima T, Shimazu T, Yamamichi N, Maekita T, Yokoi C, Oda I, Ando T, Yoshida T, Nanjo S et al: Demonstration of the usefulness of epigenetic cancer risk prediction by a multicentre prospective cohort study. Gut 2015, 64(3):388-396.

19. Dixon MF, Genta RM, Yardley JH, Correa P: Classification and grading of gastritis. The updated Sydney System. International Workshop on the Histopathology of Gastritis, Houston 1994. The American journal of surgical pathology 1996, 20(10):1161-1181.

20. Hirashita Y, Tsukamoto Y, Kudo Y, Kakisako D, Kurogi S, Hijiya N, Nakada C, Uchida T, Hirashita T, Hiratsuka T et al: Early response in phosphorylation of ribosomal protein S6 is associated with sensitivity to trametinib in colorectal cancer cells. Laboratory investigation; a journal of technical methods and pathology 2021, 101(8):1036-1047.

21. Ushijima T, Hattori N: Molecular pathways: involvement of Helicobacter pylori-triggered inflammation in the formation of an epigenetic field defect, and its usefulness as cancer risk and exposure markers. Clinical cancer research : an official journal of the American Association for Cancer Research 2012, 18(4):923-929.

22. Schlesinger Y, Straussman R, Keshet I, Farkash S, Hecht M, Zimmerman J, Eden E, Yakhini Z, BenShushan E, Reubinoff BE et al: Polycomb-mediated methylation on Lys27 of histone H3 pre-marks genes for de novo methylation in cancer. Nat Genet 2007, 39(2):232-236.

23. Nam KT, Lee HJ, Sousa JF, Weis VG, O'Neal RL, Finke PE, Romero-Gallo J, Shi G, Mills JC, Peek RM, Jr. et al: Mature chief cells are cryptic progenitors for metaplasia in the stomach. Gastroenterology 2010, 139(6):2028-2037 e2029.

24. Burclaff J, Willet SG, Sáenz JB, Mills JC: Proliferation and Differentiation of Gastric Mucous Neck and Chief Cells During Homeostasis and Injury-induced Metaplasia. Gastroenterology 2020, 158(3):598-609.e595.

25. Boyer LA, Lee TI, Cole MF, Johnstone SE, Levine SS, Zucker JP, Guenther MG, Kumar RM, Murray HL, Jenner RG et al: Core transcriptional regulatory circuitry in human embryonic stem cells. Cel/2005, 122(6):947-956.

26. Michigami Y, Watari J, Ito C, Nakai K, Yamasaki T, Kondo T, Kono T, Tozawa K, Tomita T, Oshima T et al: Long-term effects of $\mathrm{H}$. pylori eradication on epigenetic alterations related to gastric 
carcinogenesis. Scientific reports 2018, 8(1):14369.

27. Take S, Mizuno M, Ishiki K, Yoshida T, Ohara N, Yokota K, Oguma K, Okada H, Yamamoto K: The longterm risk of gastric cancer after the successful eradication of Helicobacter pylori. Journal of gastroenterology 2011, 46(3):318-324.

28. Mills JC, Shivdasani RA: Gastric epithelial stem cells. Gastroenterology 2011, 140(2):412-424.

29. Sugano K, Tack J, Kuipers EJ, Graham DY, El-Omar EM, Miura S, Haruma K, Asaka M, Uemura N, Malfertheiner $P$ et al: Kyoto global consensus report on Helicobacter pylori gastritis. Gut 2015, 64(9):1353-1367.

30. Willet SG, Lewis MA, Miao ZF, Liu D, Radyk MD, Cunningham RL, Burclaff J, Sibbel G, Lo HG, Blanc V et al: Regenerative proliferation of differentiated cells by mTORC1-dependent paligenosis. EMBO J 2018, 37(7).

31. Al-Marzoqee FY, Khoder G, Al-Awadhi H, John R, Beg A, Vincze A, Branicki F, Karam SM: Upregulation and inhibition of the nuclear translocation of Oct4 during multistep gastric carcinogenesis. International journal of oncology 2012, 41(5):1733-1743.

32. Deng N, Goh LK, Wang H, Das K, Tao J, Tan IB, Zhang S, Lee M, Wu J, Lim KH et al: A comprehensive survey of genomic alterations in gastric cancer reveals systematic patterns of molecular exclusivity and co-occurrence among distinct therapeutic targets. Gut 2012, 61(5):673-684.

33. Huang KK, Ramnarayanan K, Zhu F, Srivastava S, Xu C, Tan ALK, Lee M, Tay S, Das K, Xing M et al: Genomic and Epigenomic Profiling of High-Risk Intestinal Metaplasia Reveals Molecular Determinants of Progression to Gastric Cancer. Cancer cel/2018, 33(1):137-150 e135.

\section{Tables}

Table 1 Patient Characteristics $(n=115)$ 


\begin{tabular}{|c|c|}
\hline Characteristics & Value \\
\hline \multicolumn{2}{|l|}{$H P$-negative patients $(\mathrm{n}=11)$} \\
\hline Age (years) & $45.36 \pm 16.64$ \\
\hline Sex(female/male) & $3(27 \%) / 8(73 \%)$ \\
\hline Endoscopic atrophy* (Close/Open) & $8(100 \%) / 0(0 \%)$ \\
\hline \multicolumn{2}{|l|}{ HP-positive patients $(\mathrm{n}=29)$} \\
\hline Age (years) & $68.76 \pm 10.33$ \\
\hline Sex (female/male) & $7(24 \%) / 22(76 \%)$ \\
\hline History of gastric cancer $(-/+)$ & $16(55 \%) / 13(45 \%)$ \\
\hline Endoscopic atrophy* (Close/Open) & $6(21 \%) / 23(79 \%)$ \\
\hline \multicolumn{2}{|l|}{ Post-HPeradication patients $(n=75)$} \\
\hline Age (years) & $69.52 \pm 9.24$ \\
\hline Sex (female/male) & $26(35 \%) / 49(65 \%)$ \\
\hline Incidence of gastric cancer $(-/+)$ & $54(72 \%) / 21(28 \%) / 5$ \\
\hline$H P$ eradication therapy ** $\left(1^{\text {st }} / 2^{\text {nd }}\right)$ & $60(80 \%) / 15(20 \%)$ \\
\hline Period after eradication therapy (years) & $3.58 \pm 3.34$ \\
\hline Endoscopic atrophy* (Close/Open) & $23(35 \%) / 52(65 \%)$ \\
\hline
\end{tabular}

, *Kimura-Takemoto classification , ${ }^{* *} 1^{\text {st }}$ line treatment (PPI, [proton pump inhibitor] clarithromycin, and amoxicillin), $2^{\text {nd }}$ line treatment (PPI, metronidazole, and amoxicillin) HP, Helicobacter pylori

Table 2 Mean Pathological Features and Levels of eEF1A Dimethylation in Gastric Mucosa 
HP-negative patients $(\mathrm{n}=11) \quad$ Mean (SD)

Histological features*

\begin{tabular}{|c|c|}
\hline Activity & 0 \\
\hline Inflammation & $1.00(0.000)$ \\
\hline Atrophy & 0 \\
\hline Intestinal metaplasia & 0 \\
\hline \multicolumn{2}{|l|}{ eEF1A dimethyl level ${ }^{\star \star}$} \\
\hline Surface & $16.91(6.423)$ \\
\hline Middle & $27.68(6.010)$ \\
\hline Basal & $14.03(4.882)$ \\
\hline HP-positive patients $(\mathrm{n}=29)$ & Mean (SD) \\
\hline \multicolumn{2}{|l|}{ Histological features* } \\
\hline Activity & $0.79(0.774)$ \\
\hline Inflammation & $1.76(0.786)$ \\
\hline Atrophy & $0.55(0.736)$ \\
\hline Intestinal metaplasia & $0.07(0.371)$ \\
\hline \multicolumn{2}{|l|}{ eEF1A dimethylation level** } \\
\hline Surface & 27.95(10.954) \\
\hline Middle & 34.08 (9.658) \\
\hline Basal & $29.31(6.187)$ \\
\hline post eradicated patients $(n=75)$ & Mean (SD) \\
\hline \multicolumn{2}{|l|}{ Histological features* } \\
\hline Activity & $0.04(0.256)$ \\
\hline Inflammation & $1.20(0.637)$ \\
\hline Atrophy & $0.44(0.793)$ \\
\hline Intestinal metaplasia & $0.25(0.660)$ \\
\hline \multicolumn{2}{|l|}{ eEF1A dimethylation level** } \\
\hline Surface & $22.52(6.334)$ \\
\hline Middle & $32.59(7.437)$ \\
\hline
\end{tabular}




\begin{tabular}{ll} 
Basal & $25.26(9.700)$ \\
\hline Intestinal metaplasia & $22.01(8.86)$
\end{tabular}

*scored using the Updated Sydney System, ** immunofluorescent intensity

Table 3. Univariate and multivariate analyses of the association between dimethyl eEF1A level and GC

\begin{tabular}{|c|c|c|c|c|c|}
\hline & \multicolumn{3}{|c|}{ Univariate } & \multicolumn{2}{|l|}{ Multivariate } \\
\hline & $\mathrm{GC}-$ & $\mathrm{GC}+$ & $P$ value & $\mathrm{OR}(95 \% \mathrm{CI})$ & $P$ value \\
\hline & $(\mathrm{n}=54)$ & $(\mathrm{n}=21)$ & & - & - \\
\hline Age (years, $<70 / \geq 70$ ) & $26 / 28$ & $8 / 13$ & 0.4323 & - & - \\
\hline Sex (female/male) & $19 / 35$ & $7 / 14$ & 0.8795 & - & - \\
\hline Period after eradication (years, $<3.5 / \geq 3.5$ ) & $32 / 22$ & $11 / 10$ & 0.5895 & & \\
\hline Histological activity (high/low) & $53 / 1$ & $20 / 1$ & 0.5054 & - & - \\
\hline Histological inflammation (high/low) & $41 / 13$ & $14 / 7$ & 0.4219 & - & - \\
\hline Histological atrophy (high/low) & $48 / 6$ & $11 / 10$ & $0.0009^{* \star}$ & $9.9493(1.7096-57.901)$ & $p=0.0106^{*}$ \\
\hline Intestinal metaplasia & $47 / 7$ & $17 / 4$ & 0.5127 & - & - \\
\hline Dimethyl-eEF1A in basal area (low/high) & $37 / 17$ & $6 / 15$ & $0.0036^{\star}$ & $3.6611(1.0350-12.949)$ & $p=0.0441^{*}$ \\
\hline
\end{tabular}

$G C$ gastric cancer, $O R$ odds ratio, $C I$ confidence interval

\section{Figures}


Figure 1

a

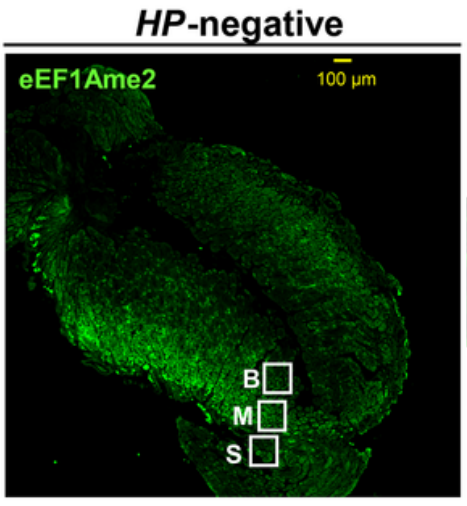

b
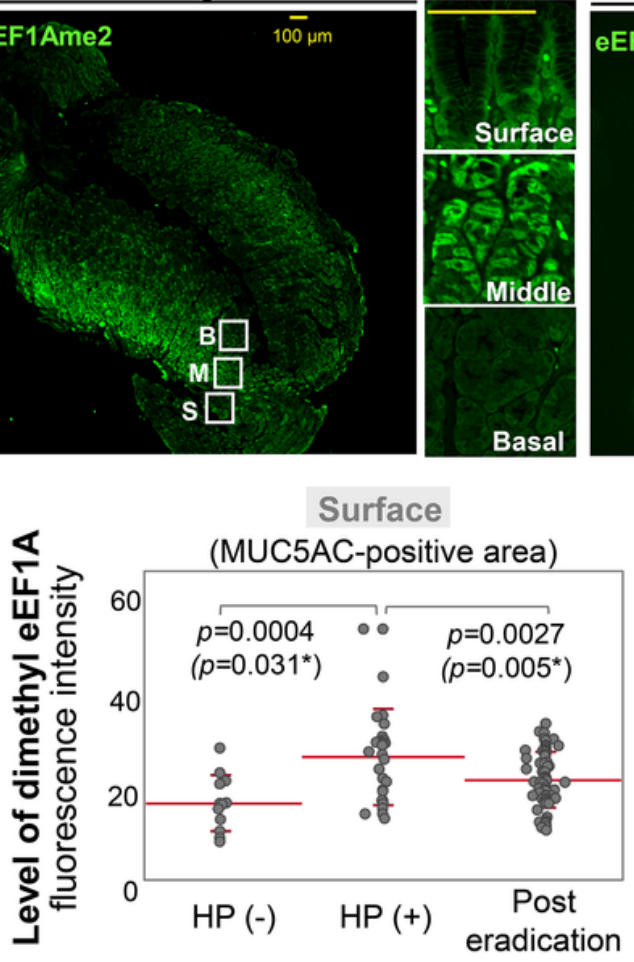

Basal
HP-positive

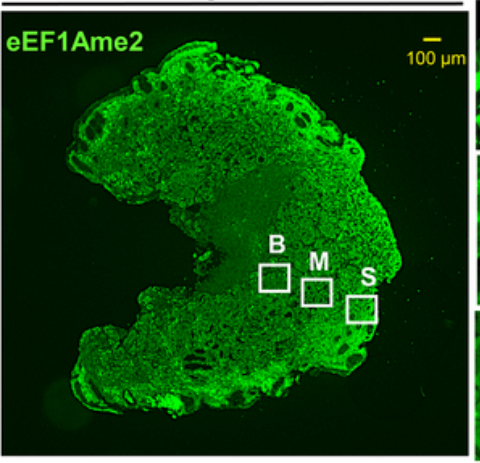

Middle

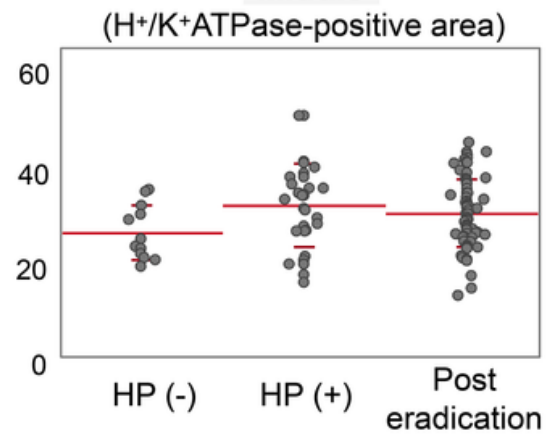

Post-eradication therapy
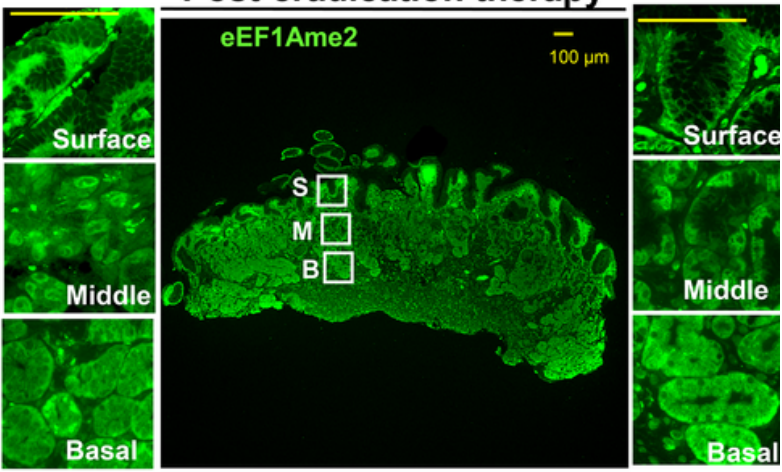

Basal

(PG1-positive area)

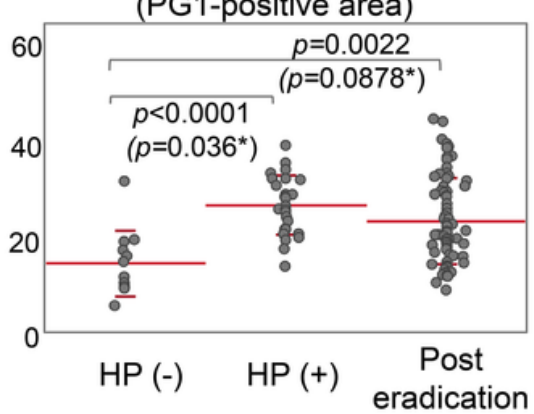

Figure 1

Immunofluorescent staining of dimethyl-eEF1A in $\mathrm{H}$. pylori-negative, -positive, and -eradicated gastric mucosa. (a) Representative images of immunofluorescent staining using an antibody against the level of dimethyl-eEF1A (eEF1AK55me2). Surface, middle, and basal areas were defined by co-staining with markers specific for each area (see Supplementary Figure 1). (b) Levels of dimethyl-eEF1A in surface, middle, and basal areas were compared between $\mathrm{H}$. pylori-negative, -positive, and post-eradicated patients. Differences were calculated by Tukey test. ${ }^{\star} p<0.05 ; * \star p<0.01 ; * \star \star p<0.001$ 


\section{Figure 2}

a
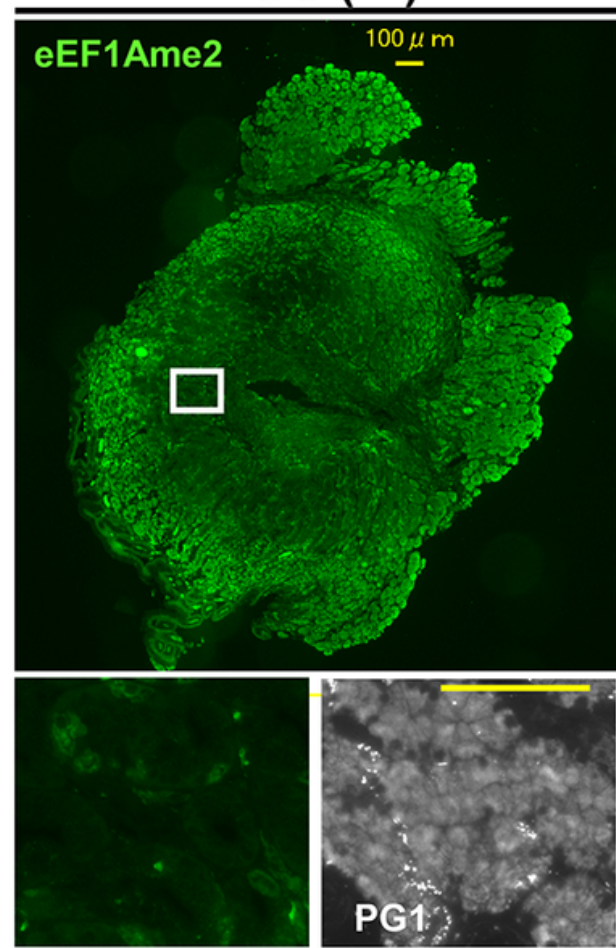

GC (-)

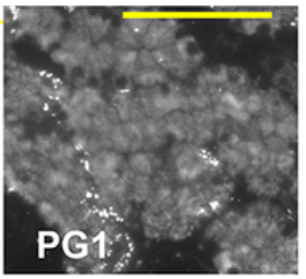

GC (+)
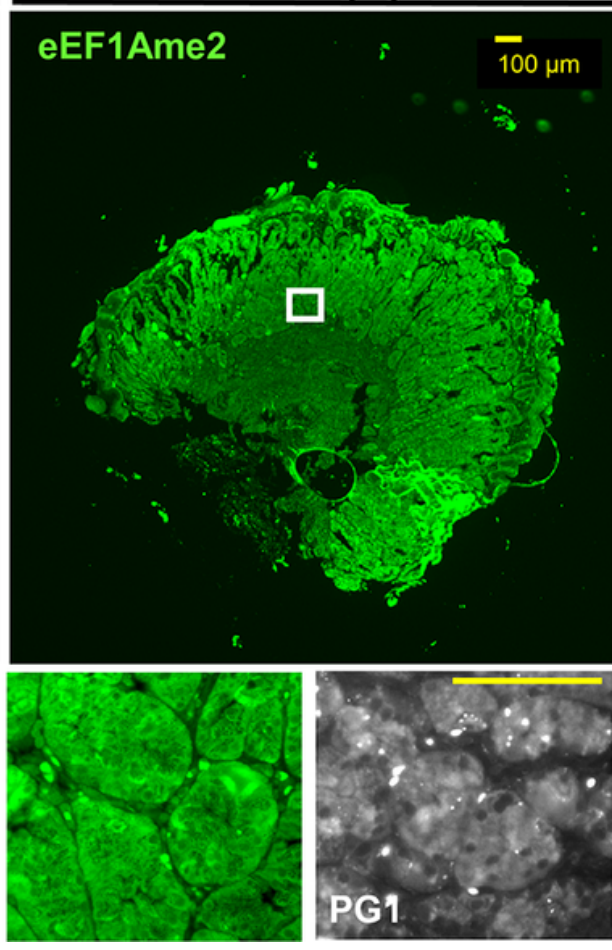

b

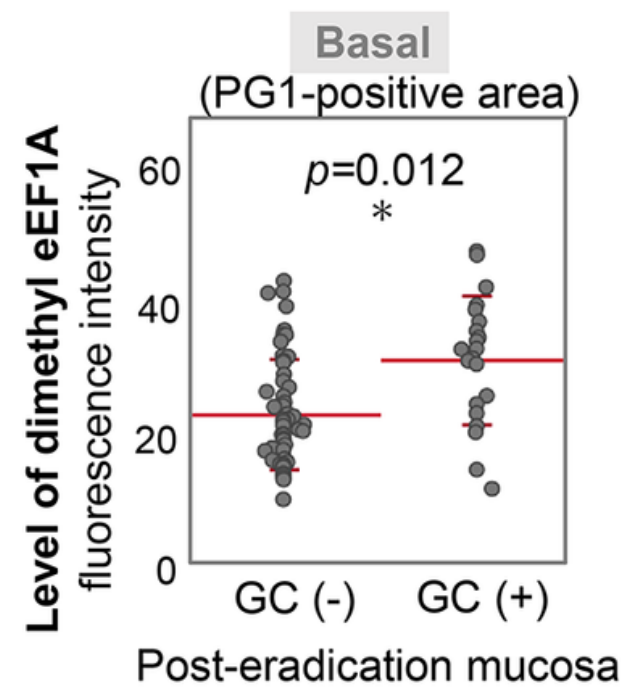

Figure 2

Immunofluorescent staining of dimethyl-eEF1A in H. pylori-eradicated mucosa with or without GC incidence. (a) Representative images of immunofluorescent staining using an antibody against the level of dimethyl-eEF1A (eEF1AK55me2) and PG-1 for H. pylori-eradicated gastric mucosa. (b) Levels of dimethyl-eEF1A in basal areas were compared between post-eradication patients with or without GC incidence. Differences were calculated by Mann-Whitney U test. ${ }^{*} p<0.05 ;{ }^{* \star} p<0.01 ;{ }^{* \star} p<0.001$ 


\section{Figure 3}
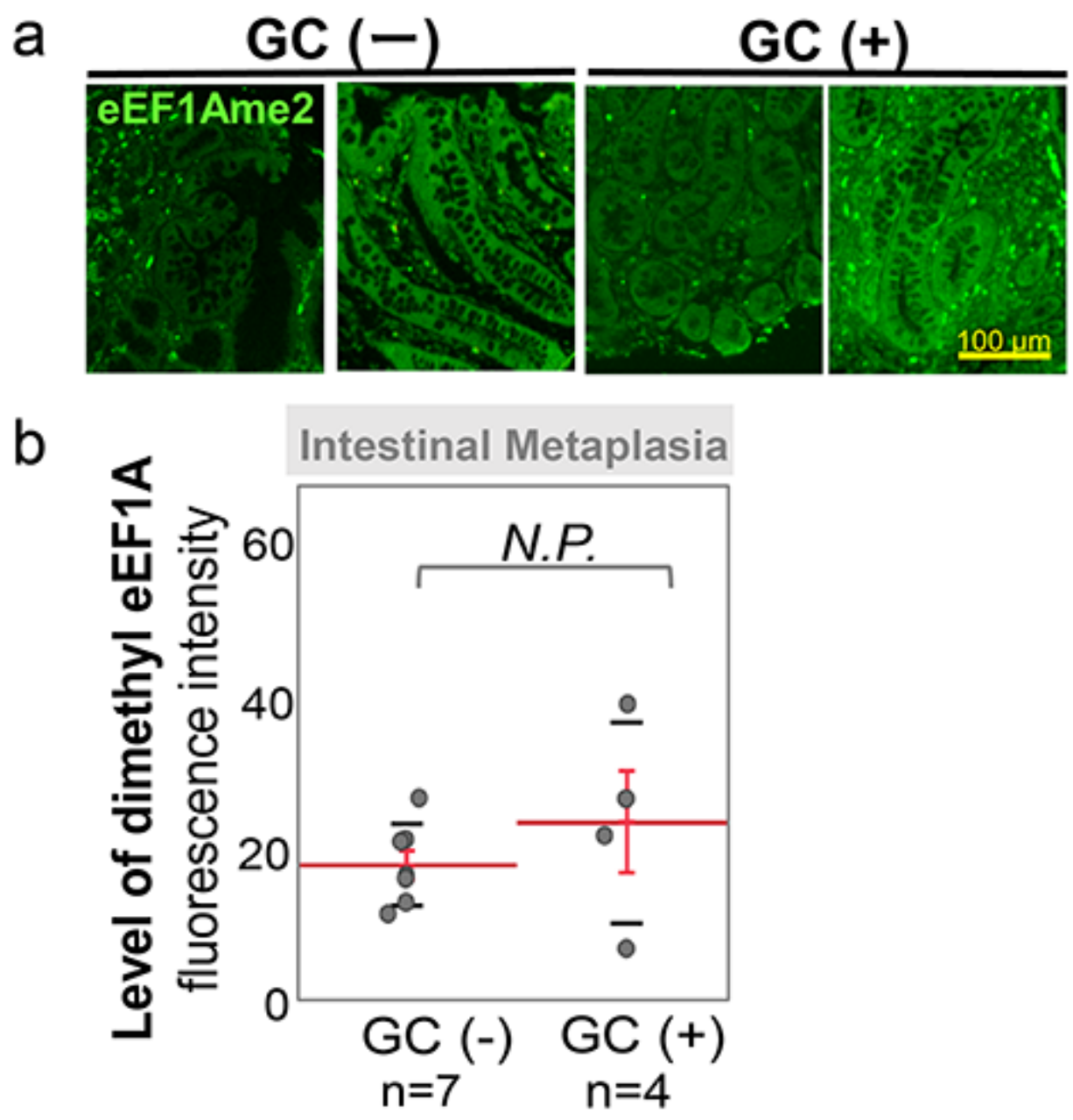

Figure 3

Comparison of dimethyl-eEF1A in an area of intestinal metaplasia with or without GC incidence. (a) Representative images of immunofluorescent staining using an antibody against the level of dimethyleEF1A (eEF1AK55me2) for eradicated gastric mucosa. (b) Levels of dimethyl-eEF1A were compared between patients with or without an incidence of GC using the Mann-Whitney $U$ test 
Figure 4

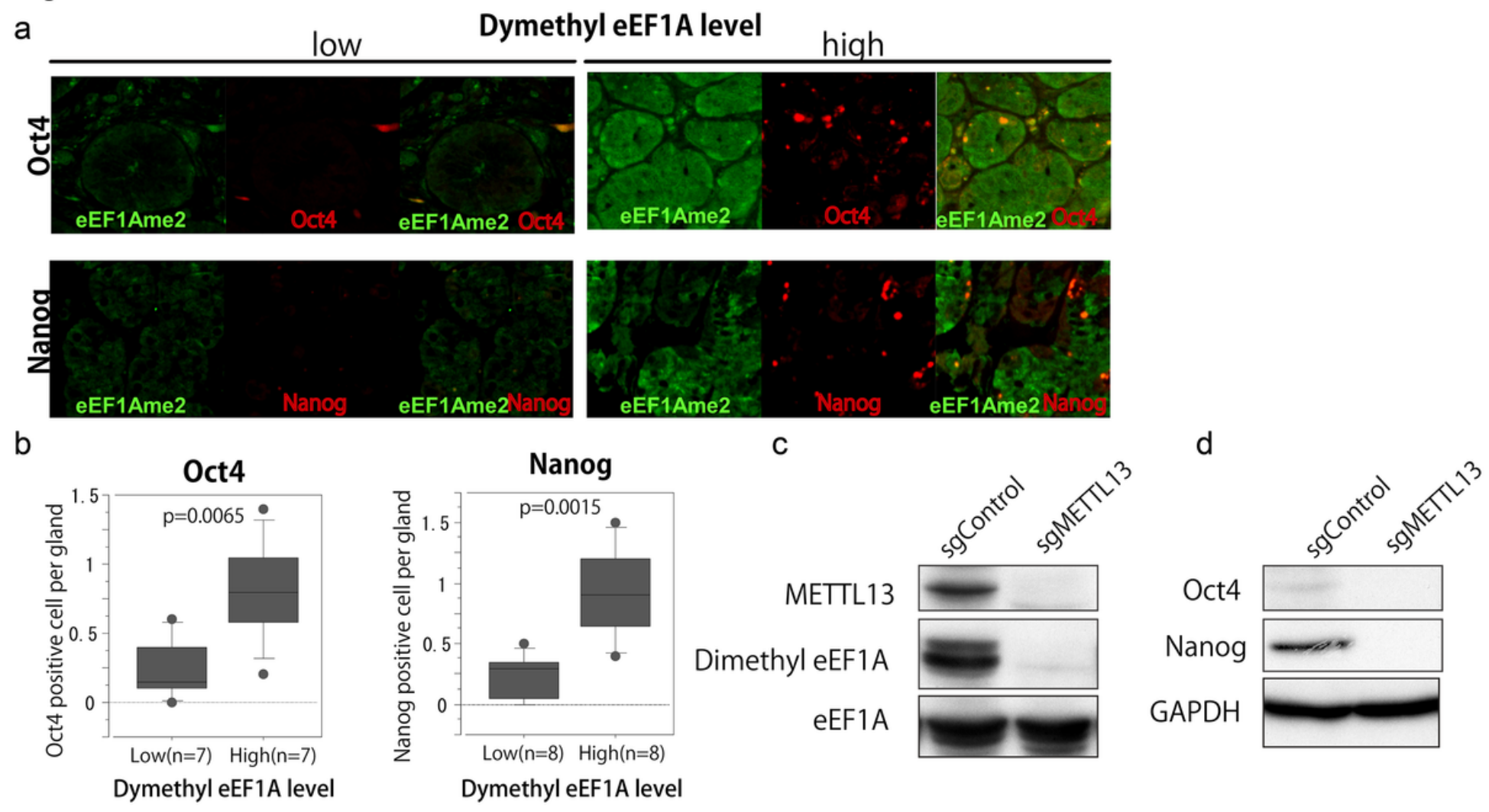

Figure 4

Dimethyl-eEF1A were compared with expressions of Oct4 and Nanog in eradicated tissues and Mettl13deleted cell. (a) Representative images of immunofluorescent staining using antibodies against dimethyleEF1A, Oct4, and Nanog in H. pylori-eradicated mucosa. (b) The numbers of Oct4- or Nanog-positive cells per gland in $\mathrm{H}$. pylori-eradicated mucosa were compared between patients with low levels (Oct4: $n=7$, Nanog: $n=8$ ) and those with high levels (Oct4: $n=7$, Nanog: $n=8$ ) of eEF1A dimethylation by MannWhitney $U$ test. (c) Knockout of Mettl13 and downregulation of eEF1A dimethylation in TMK-1 cells were confirmed by Western blotting. (d) Western blotting with antibodies against Oct4, Nanog, and GAPDH revealed that knockout of Mettl13 in TMK-1 cell caused downregulation of Oct4 and Nanog expressions

\section{Supplementary Files}

This is a list of supplementary files associated with this preprint. Click to download.

- Suppfigure1.tif

- Suppfigure2.tif 